\title{
Demographic variation in fit note receipt and long- term conditions in south London
}

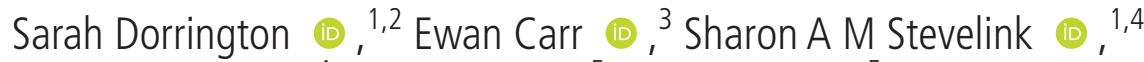 \\ Alexandru Dregan, ${ }^{1}$ David Whitney, ${ }^{5}$ Stevo Durbaba, ${ }^{5}$ Mark Ashworth, ${ }^{5}$ \\ Arnstein Mykletun, ${ }^{6,7,8,9,10}$ Matthew Broadbent, ${ }^{2}$ Ira Madan (10 , ${ }^{11}$ \\ Stephani Hatch (1) , Matthew Hotopf (1) 1,2
}

- Additional material is published online only. To view please visit the journal online (http://dx.doi.org/10.1136/ oemed-2019-106035).

For numbered affiliations see end of article.

\section{Correspondence to} Dr Sarah Dorrington, Institute of Psychiatry, Psychology \& Neuroscience, King's College London, London SE5 8AF, London, UK; sarah.dorrington@kcl.ac.uk

Received 20 June 2019 Revised 2 December 2019 Accepted 14 February 2020 Published Online First 24 March 2020

\section{ABSTRACT \\ Objectives Introduced in the UK in 2010, the fit note was designed to address the problem of long-term sickness absence. We explored (1) associations between demographic variables and fit note receipt, 'maybe fit' use and long-term conditions, (2) whether individuals with long-term conditions receive more fit notes and are more likely to have the 'maybe fit' option selected and (3) whether long-term conditions explained associations between demographic variables and fit note receipt. Methods Data were extracted from Lambeth DataNet, a database containing electronic medical records of all 45 general practitioner (GP) practices within the borough of Lambeth. Individual-level anonymised data on GP consultations, prescriptions, Quality and Outcomes Framework diagnostic data and demographic information were analysed using survival analysis.}

Results In a sample of 326415 people, 41502 $(12.7 \%)$ received a fit note. We found substantial differences in fit note receipt by gender, age, ethnicity and area-level deprivation. Chronic pain (HR 3.7 (95\% Cl 3.3 to 4.0)) and depression (HR $3.4(95 \% \mathrm{Cl} 3.3$ to 3.6)) had the highest rates for first fit note receipt. 'Maybe fit' recommendations were used least often in patients with epilepsy and serious mental illness. The presence of longterm conditions did not explain associations between demographic variables and fit note use.

Conclusions For the first time, we show the relationships between fit note use and long-term conditions using individual-level primary care data from south London. Further research is required in order to evaluate this relatively new policy and to understand the needs of the population it was designed to support.

\section{INTRODUCTION}

Long-term sickness absence has damaging consequences for affected individuals and families leading to social exclusion, adverse health outcomes and financial insecurity. ${ }^{1}$ In 2008, a United Kingdom (UK) government report suggested that the process of sickness certification by general practitioners (GPs) was a contributor to the problem of long-term sickness absence. ${ }^{2}$ In the UK, sickness certification is most often delivered to an unwell employee by a GP, after 7 days or more sickness absence. ${ }^{3}$ The fit note, introduced in 2010 in primary care in the UK, was designed to address the problem of longterm sickness absence by changing the sickness

\section{Key messages}

What is already known about this subject?

- The fit note was designed to address the problem of long-term sickness absence by changing the sick note from a binary fit/not fit to include the third option: 'maybe fit' with associated work adjustments.

- Previous research has found fit note use to be highest among women, patients with mental health conditions and those with higher levels of deprivation. Work adjustments are recommended more often for physical conditions and for individuals with lower levels of deprivation.

\section{What are the new findings?}

- We explored associations between fit note use and long-term conditions, using clinical records, rather than being limited to clinical information provided on the fit note.

- Patients with chronic pain and depression had the highest rates for first fit note receipt. 'Maybe fit' recommendations were used least often in patients with epilepsy and serious mental illness.

- We found wide demographic variation in prevalence of long-term conditions and risk of fit note receipt. Long-term conditions did not explain demographic variation in fit note use, suggesting that additional factors, such as job type, may contribute to fit note use.

\section{How might this impact on policy or clinical practice in the foreseeable future? \\ - An understanding of the underlying needs of workers presenting for a fit note is necessary to enable policymakers and clinicians to support the working age population.}

certification process from advising individuals on their inability to work to advising them on what they could do if work could be adapted. ${ }^{4}$ The fit note expands the 'fit to work' or 'not fit to work' options on the sick note, by including a third 'maybe fit' option. 'Not fit for work' and the new 'maybe fit' option both provide evidence of sickness to an employer and enable individuals to access sickness 
benefits. However, the 'maybe fit' option encourages GPs to recommend work adjustments, such as reduced hours or altered duties. Each fit note is prescribed for a period of time decided by the issuing GP; this may be for days, weeks or months. The GP can extend the period of sickness absence prescribed on the first fit note (FFN) by providing further fit notes, each valid for a specified period of time. The 'fit to work', 'not fit to work' or 'maybe fit' option chosen when the fit note is prescribed applies to the worker's presentation at that time, and it may change on future fit notes according to the factors such as illness severity and employment type. The 'maybe fit' option is similar to partial sickness absence in Nordic countries but does not have the same legislative support. ${ }^{5}$ In the UK there is no legal obligation for employers to observe GP recommendations or for GPs to recommend adjustments to workers.

Despite the scale and importance of the fit note policy, there has been surprisingly little independent research into fit note use. ${ }^{6}$ Previous studies have either used survey methods (where participation is inevitably incomplete leading to selection bias), ${ }^{7}$ aggregated routine data (which suffer from the ecological fallacy, when an inference is made about an individual based on aggregate data for a group) ${ }^{8}$ or relied on information collected directly from fit notes, with a lack of information on long-term conditions and individual-level demographic variables. ${ }^{9}$

The largest study of fit note use, a national study by National Health Service (NHS) Digital, analysed over 5 million fit notes issued between 2014 and 2017: results were not analysed at the individual level, but were aggregated by Clinical Commissioning Groups (geographically based commissioning boards which operate across the NHS), and analysed using diagnosis and date of issue. ${ }^{8}$ Consistent with the findings of the NHS Digital study, our systematic review of fit note research found fit note use to be highest among women, people requesting sickness certification for a mental health condition and more deprived individuals. ${ }^{6}$ Work adjustments were recommended more often to the groups least likely to receive a fit note: those requesting sickness certification for physical conditions and individuals with lower levels of deprivation. ${ }^{6}$ Department of Work and Pensions fit note guidelines published in 2013 advised GPs to provide fit notes for shorter periods of time, for workers who aimed to return to modified work and for patients who were likely to need to remain on sick leave. In practice, fit note use varies widely by GP practice and by GPs' occupational health training. ${ }^{9}{ }^{10}$ There is some evidence of positive feedback from employers in response to clear work adjustment recommendations from GPs. ${ }^{6}$

Our aim in this study was to describe fit note use in the primary care population of Lambeth, an inner-city borough in south London characterised by an ethnically and socioeconomically diverse population. The present study explored (1) associations between demographic variables and fit note receipt, 'maybe fit' use and long-term conditions, (2) whether individuals with longterm conditions receive more fit notes and are more likely to have the 'maybe fit' option selected and (3) whether long-term conditions explained associations between demographic variables and fit note receipt.

\section{METHODS}

\section{Data}

This is a longitudinal study using prospective data from electronic medical records. The duration of data gathering dates back to the introduction of electronic health records (digitisation) in the study practices. This process was gradual but all practices in Lambeth had introduced full electronic health records by January 2005; most had completed this process 3-5 years earlier. Data were extracted from the electronic medical records of all 45 general practices within the London Borough of Lambeth, as part of Lambeth DataNet (LDN). LDN is a primary care database which collects individual-level anonymised data on clinical diagnoses, referrals, consultations, treatment prescriptions, lab tests and public health initiatives, such as the Quality and Outcomes Framework (QOF) diagnostic data. LDN also includes demographic information such as age, gender and self-identified ethnicity. LDN was created in 2006 as a local resource to improve ethnicity recording and to explore local health inequalities, particularly those based on ethnicity or social deprivation. ${ }^{11}{ }^{12}$ The London Borough of Lambeth, home to a population of just over 318,000 , is the 22 nd most deprived local authority in England ${ }^{13}$ and has the 4th highest level of income inequality of any borough in London. ${ }^{14}$ Lambeth contains a young and ethnically diverse population, including large Portuguese, South American and Black populations. ${ }^{15}$ Around 150 different languages are spoken and around 17000 people (6\% of the population) speak a main language other than English. ${ }^{13}$ Thirty-eight per cent of the population was born outside of the UK. ${ }^{13}$ The number of individuals registered on LDN at any one time $(n=405000)$ exceeds the total Lambeth population recorded in the Census due to the cross-boundary registration of patients not living in Lambeth, GP list inflation and the temporary residence of students in the Lambeth area from surrounding universities. We analysed records covering a period of 40 months from 1 January 2014 to 30 April 2017. Data were extracted in May 2017 from the primary care clinical record system, EMIS Web. We restricted the sample to working-age adults aged 16-60 years in order to prevent exit from the workforce due to reaching statutory pension age (60-65 years during our study period), which is not measured in our sample.

\section{Measures}

\section{Demographic variables}

Information on individuals' first recorded sex, year of birth, ethnicity and 2011-defined lower super output area (LSOA, an area covering an average population of $1722^{16}$ ) were extracted from the GP records. Ethnicity was coded using seven subcategories of ethnicity (Asian, Black African, Black Caribbean, BlackOther, Other,Mixed, and White). Following previous research within south London that suggested differences in mental health outcomes between Black Caribbean and Black African groups, the same classification was used in this study, as defined in the 2011 Census. ${ }^{1718}$ LSOA data was used to derive a measure of deprivation for each individual based on area of residence and the Index of Multiple Deprivation (IMD; 2010, after conversion to 2011 LSOA values). IMD scores were divided into quintiles, based on deprivation scores in Lambeth.

\section{Long-term conditions and pain}

Presence of long-term conditions was assessed using the QOF data, which is an annual reward and incentive programme for all GP practices in England. It requires GPs to identify individuals who meet quality targets for various long-term conditions such as depression, obesity and diabetes. We included in our analysis binary indicators (present/not present) of $15 \mathrm{QOF}^{19}$ conditions: depression, epilepsy, diabetes mellitus, coronary heart disease (CHD), chronic obstructive pulmonary disorder (COPD), cancer (non-specified), atrial fibrillation (AF), heart failure (HF), stroke, rheumatoid arthritis, obesity, serious mental illness (SMI: schizophrenia and bipolar disorder), learning 
Table 1 Distribution of LTC in Lambeth Datanet by gender, age, self-defined ethnicity and IMD

\begin{tabular}{|c|c|c|c|c|c|c|}
\hline \multicolumn{2}{|l|}{ ICD-10 chapter } & \multirow{2}{*}{$\begin{array}{l}\text { n/a } \\
\text { Any LTC }\end{array}$} & \multirow{2}{*}{4} & \multirow{2}{*}{5} & \multirow{2}{*}{$\begin{array}{l}9 \\
\text { Cardiovascular disease* } \\
\%\end{array}$} & \multirow{2}{*}{$\begin{array}{l}10 \\
\text { Respiratory diseaset } \\
\%\end{array}$} \\
\hline & Number (\%) & & & & & \\
\hline Demographic & $n=326415$ & $n=113979$ & $n=49847$ & $n=33477$ & $n=19605$ & $n=35153$ \\
\hline$\%$ population & 100.0 & 34.9 & 15.3 & 10.3 & 6.0 & 10.8 \\
\hline \multicolumn{7}{|l|}{ Gender } \\
\hline Male & $153228(46.9)$ & 33.9 & 13.4 & 8.5 & 6.8 & 10.8 \\
\hline Female & $173187(53.1)$ & 35.8 & 16.9 & 11.8 & 5.3 & 10.8 \\
\hline \multicolumn{7}{|l|}{ Age (years) } \\
\hline $16-20$ & $12945(4.0)$ & 20.9 & 6.5 & 4.4 & 0.3 & 11.1 \\
\hline $21-25$ & $52933(16.2)$ & 23.4 & 6.6 & 6.1 & 0.4 & 11.9 \\
\hline $26-30$ & 72165 (22.1) & 24.8 & 8.0 & 7.6 & 0.7 & 10.9 \\
\hline $31-35$ & $58175(17.8)$ & 28.8 & 11.3 & 9.4 & 1.6 & 10.0 \\
\hline $36-40$ & $40271(12.3)$ & 35.5 & 16.5 & 11.5 & 3.7 & 9.4 \\
\hline $41-45$ & 30295 (9.3) & 45.3 & 23.1 & 14.0 & 8.6 & 9.9 \\
\hline $46-50$ & $25690(7.9)$ & 55.0 & 29.5 & 15.8 & 16.0 & 11.0 \\
\hline $51-55$ & 20041 (6.1) & 62.2 & 33.7 & 17.2 & 24.7 & 11.9 \\
\hline $56-60$ & $13900(4.3)$ & 69.1 & 36.8 & 17.3 & 34.7 & 12.6 \\
\hline \multicolumn{7}{|l|}{ Ethnicity } \\
\hline White & $220205(67.5)$ & 31.5 & 11.3 & 10.7 & 3.7 & 11.4 \\
\hline Black African & $30512(9.4)$ & 47.8 & 31.6 & 7.5 & 15.6 & 6.4 \\
\hline Asian & $24145(7.4)$ & 30.3 & 12.1 & 6.7 & 6.6 & 8.9 \\
\hline Black Caribbean & $16845(5.2)$ & 58.5 & 34.5 & 14.6 & 17.3 & 14.8 \\
\hline Mixed & $15933(4.9)$ & 36.6 & 16.3 & 11.2 & 5.5 & 11.6 \\
\hline Other & 11144 (3.4) & 29.1 & 16.0 & 7.9 & 4.5 & 5.4 \\
\hline Black Other & $7631(2.3)$ & 50.5 & 28.9 & 12.0 & 11.9 & 12.6 \\
\hline \multicolumn{7}{|l|}{ IMD quintile } \\
\hline 1 Least deprived & $65367(20.0)$ & 31.5 & 11.8 & 8.8 & 4.8 & 11.3 \\
\hline 2 & 64851 (19.9) & 32.9 & 13.2 & 9.7 & 5.1 & 11.0 \\
\hline 3 & $67651(20.7)$ & 34.1 & 14.3 & 10.5 & 5.6 & 10.7 \\
\hline 4 & $63672(19.5)$ & 37.2 & 17.4 & 11.2 & 7.2 & 10.5 \\
\hline 5 Most deprived & $64874(19.9)$ & 39.1 & 19.7 & 11.2 & 8.5 & 10.4 \\
\hline
\end{tabular}

${ }^{*}$ Coronary heart disease, atrial fibrillation, heart failure, peripheral arterial disease, stroke, transient ischaemic attack and hypertension.

†Asthma and COPD.

COPD, chronic obstructive pulmonary disorder; ICD-10, International Classification of Diseases 10; IMD, Index of Multiple Deprivation; LTC, long-term condition.

disability, hypertension and asthma. In addition, chronic pain ${ }^{20}$ was derived based on receipt (yes/no) of any prescriptions listed in British National Formulary medication chapters 4.7.2 or 4.7.3 (with repeat, repeat dispensed or automatic issue type). QOF conditions excluded from our analysis were smoking, palliative care (patients are very unlikely to be in employment), osteoporosis, dementia and chronic kidney disease (excluded due to the small numbers with these diagnoses in the age group under study).

QOF measures have been shown to underestimate prevalence of disorders in the general population due to the reliance on presentation to the GP for diagnosis. ${ }^{21}$ QOF diagnosis is therefore a specific, but not sensitive measure ${ }^{22}$ with a low falsepositive but high false-negative rates. In this paper we applied QOF35 criteria, published by the Department of Health in October 2017. Online supplementary table 5 describes the definition of each QOF diagnosis included.

Where possible, QOF diagnoses were grouped according to International Classification of Diseases 10 (ICD-10) chapter groupings (Table 1 and supplementary table 1). 'Respiratory' includes asthma and COPD; 'cardio' includes CHD, AF and HF; 'vascular' includes stroke and peripheral arterial disease (PAD) and 'cardiovascular' includes $\mathrm{CHD}, \mathrm{AF}, \mathrm{HF}$, stroke, $\mathrm{PAD}$ and hypertension.

\section{Fit note}

Date of FFN was derived for each participant based on digital fit notes issued between January 2014 and May 2017. We excluded fit notes issued before this period because although digital fit notes were introduced in 2012, they were not in widespread use until 2014. Due to the lack of information on prior sickness certification (before January 2014), we could not determine whether a 'FFN' during the study period represented the individual's first ever sickness certification. The mean number of fit notes received per person within the 40 -month period was analysed by demographic variables. Within the population given a fit note, every fit note has the potential to be classified as 'maybe fit'. Each fit note which recommended the 'maybe fit' option was categorised as ' 1 ' ('maybe fit to work') versus ' 0 ' ('not fit to work' or 'fit to work') to create an indicator of receipt of 'maybe fit' note.

\section{Statistical analysis}

Descriptive statistics (ie frequency, percentage, mean) were used to describe baseline characteristics among study patients. Risk of receiving a fit note over the 40 -month period was analysed using multi-level Cox regression, taking into account the clustering 
of patients within GP practices. We included in our analyses each patient's FFN during the study period. Time at risk of a fit note was calculated from the beginning of the study period (1 January 2014) or registration with a GP practice in Lambeth (whichever occurred later), until one of four events occurred: receipt of fit note, deregistered from Lambeth primary care, date of death or end-of-study period (30 April 2017). The latter three events (discharge, date of death and end of study) were treated as censoring events. The proportional hazards assumption was assessed (via Schoenfeld Residuals and visual inspection of Kaplan-Meier curves) and found not to be violated. The analyses adjusted for all study covariates, including demographic variables (age, gender and LSOA deprivation) and chronic disorders (eg, depression and cardiovascular conditions). The population attributable fraction (PAF) was calculated using the Miettinen formula. ${ }^{23}$ The PAF is a way of estimating the proportion of the incidence of fit note receipt in the population that is potentially attributable to 'exposure' to each long-term condition. Within the sample who received a fit note, the odds of 'maybe fit' use were analysed using logistic regression, also taking into account the clustering of patients within GP practices. All models were estimated using Stata V.15 (Stata Corporation).

\section{RESULTS}

The analytical sample included 326415 people, after removing 76497 individuals with missing information on ethnicity $(13.1 \%$ $n=42716)$ or IMD $(6.8 \% n=22282)$ or both $3.5 \% n=11499)$.
Compared with the analytical sample, excluded individuals (online supplementary table 3 ) were younger $(17.6 \%$ vs $4.0 \%$ aged $16-20)$, more likely to be men (54.8\% vs $46.9 \%)$, less likely to report at least one type of long-term condition $(28.0 \%$ vs $34.9 \%)$ and slightly less likely to have received a fit note $(11.9 \%$ vs $12.7 \%)$. Demographic characteristics of the population are shown in table 1 . The largest age groups were those aged 26-30 years $(22.1 \%)$ and $31-35$ years $(17.8 \%)$, with higher proportion of women relative to men (53.1\% and $46.9 \%$, respectively). The largest ethnic group was White (67.5\%), followed by Black African (9.4\%).

\section{Demographic differences in fit note receipt}

Fit notes were received by 41502 people, $12.7 \%$ of the working age population. Table 2 presents the risk of FFN receipt by demographic variables. Risk of FFN was greater among women than men (HR 1.30, 95\% CI (1.26 to 1.35)). People aged 16-40 had a similar risk of fit note receipt, but the risk increased thereafter, with the highest risks being associated with the oldest age groups. Living in an area with higher deprivation was associated with increased risk of fit note receipt (HR 1.68, 95\% CI (1.49 to 1.90$)$ ). These findings persisted after mutually controlling for all demographic variables (partially adjusted model) and longterm conditions (fully adjusted model), though the associations between FFN and gender, age, ethnic group and deprivation were somewhat attenuated.

Table 2 Fit note receipt in Lambeth Datanet by gender, age, self-defined ethnicity and IMD

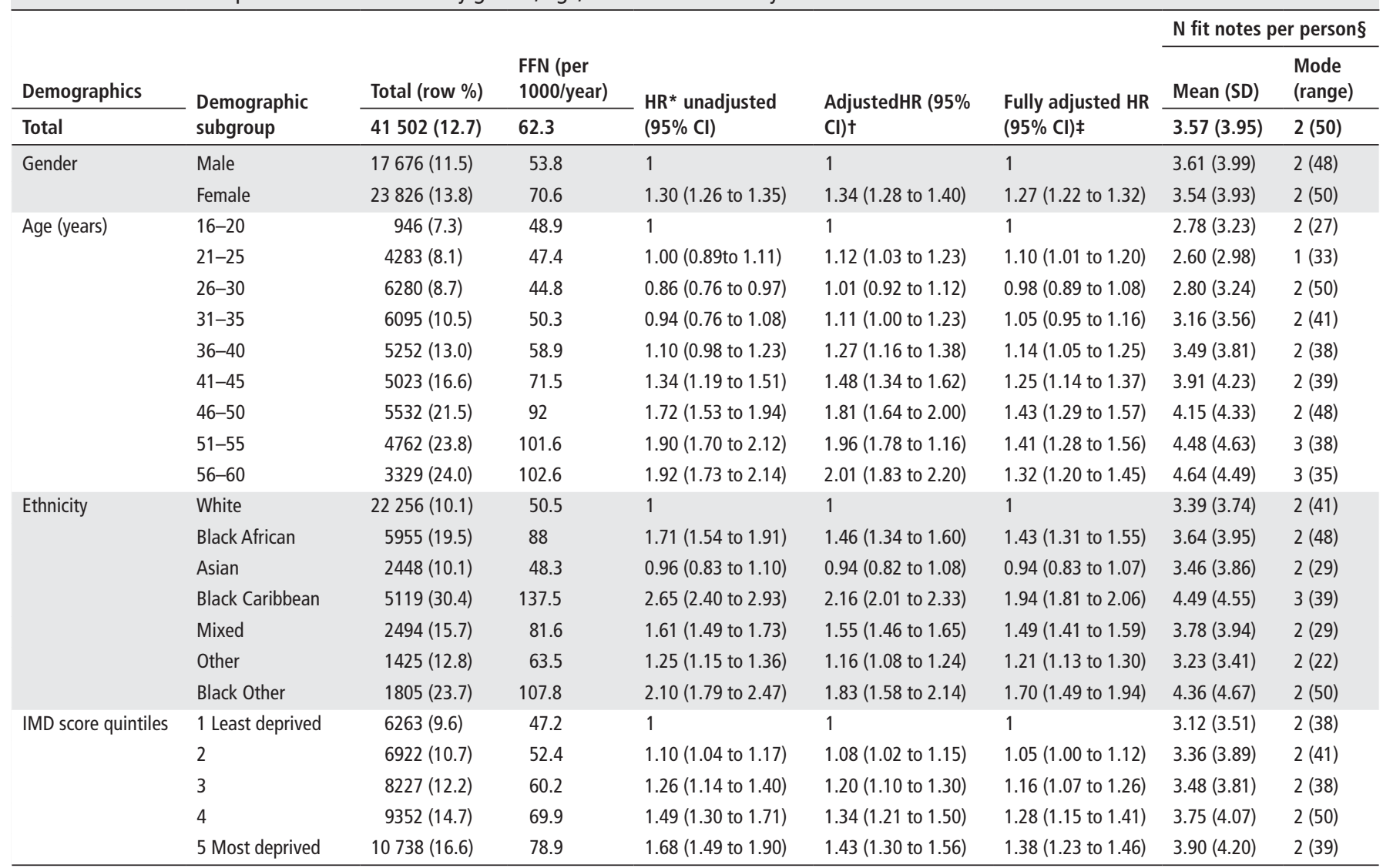

\footnotetext{
*Hazard ratios estimate a ratio of time to FFN for each demographic group compared with the first group in each category.
}

†Adjusted for age, gender, ethnicity and IMD score.

$\ddagger$ Adjusted for age, gender, ethnicity, IMD score and having a long-term condition.

$\S$ In population with a fit note.

FFN, first fit note; HR, Hazard Ratio; IMD, Index of Multiple Deprivation; QOF, Quality and Outcomes Framework. 
When looking at all fit notes, rather than the FFN, we found that the mean number of fit notes per person also varied by age, ethnicity and deprivation. Older individuals, those representing Black Caribbean, Black-Other or Mixed ethnic groups, and the most deprived quintile received on average more fit notes. Regarding the type of fit note received, the 'maybe fit' option was recommended to $6139(14.8 \%)$ of people receiving a fit note (table 3). 'Maybe fit' was more commonly recommended to women (HR 1.10, 95\% CI (1.04 to 1.16)), the Black Caribbean group (1.14, 95\% CI 1.04 to 1.25$)$ ), and people over 20 years of age. No association emerged between area deprivation and use of the 'maybe fit' option. There was no difference in 'maybe fit' use by deprivation.

\section{Demographic differences in diagnosis of long-term conditions}

Table 1 and online supplementary table 1 show the distribution of long-term conditions by demographic variables. Women presented with a higher prevalence of any long-term condition compared with men (53.1\% vs 46.9\%). Prevalence of long-term conditions increased with age. The ethnic groups with low fit note use (Asian and Other and White) were less likely to have a long-term condition than the Black African, Black Caribbean Black-Other and Mixed ethnic groups. Patients with missing ethnicity were least likely to have a QOF diagnosis. There was a linear association between long-term conditions and living in a more deprived area. The three most common long-term conditions were obesity $(15.3 \%)$, respiratory disease $(10.8 \%)$ and depression (10.3\%). Individual long-term conditions showed somewhat different patterns with demographic variables. For example, while cardiovascular disease, obesity and depression became more prevalent with increasing age, respiratory disease was common across age groups. The full list of long-term conditions by demographic distribution is shown in online supplementary table 1 .

\section{Long-term conditions and risk of fit note receipt}

Table 4 shows the risk of FFN receipt by diagnostic groups, stratified by age groups. Overall, individuals with a long-term condition were at greater risk of receiving a fit note than those without. After adjustment for age, sex, ethnicity and area deprivation, for virtually every age group, presence of a long-term condition was associated with a twofold to threefold increase in fit note receipt compared with those without. In the group aged 16-40 years, the highest risk of fit note receipt was among people with chronic pain, depression, vascular disease and SMI. In the older age groups, risk of FFN receipt was highest among people with depression, chronic pain, rheumatoid arthritis and vascular disease. In the fully adjusted model, chronic pain (HR $3.7,95 \%$ CI (3.3 to 4.0$)$ ) and depression (3.4, 95\% CI (3.3 to 3.6)) had the highest HR for FFN receipt.

The final column in table 4 shows the PAF of long-term conditions. Depression had the highest PAF for fit note use overall (17.5\%) and for younger age groups (15.3\% age 16-40, 19.6\% age 41-50). Obesity had the highest PAF for people age 51-60 $(23.0 \%)$.

Table 5 shows the odds of receiving a 'maybe fit' recommendation among individuals who received a fit note. Use of the 'maybe fit' option was lower among individuals with epilepsy (HR $0.73,95 \%$ CI $(0.56$ to 0.94$)$ ) and serious mental illness (HR 0.53 , 95\% CI $(0.43$ to 0.66$)$ ) than people with no longterm conditions. For the remaining 11 diagnoses, there was no association between diagnosis and use of the 'maybe fit' option.

\section{DISCUSSION}

For the first time, we show the associations between fit note receipt and long-term conditions using individual-level data in a large and diverse population. We found substantial differences in fit note receipt by gender, age, ethnicity and area-level deprivation. While positive associations between fit note receipt, age and deprivation are to be expected, the association of fit note receipt with ethnicity requires further explanation. Even after accounting for differences in long-term conditions and arealevel deprivation, all minority ethnic groups except the Asian group experienced increased fit note receipt. Evidence suggests that socioeconomic inequalities are drivers of health inequalities, rather than inequalities in access to primary care. ${ }^{24} 25$ Area-level deprivation is a proxy measure of individual deprivation levels, if individual-level socioeconomic status had been available differences between ethnic groups may have been further attenuated. Similarly, we relied on diagnosis of QOF long-term conditions when other causes of ill health may have been driving the differences between ethnic groups, for example mental health symptoms which do not meet criteria or threshold for long-term conditions such as low mood or stress.

Fit note receipt is likely to be influenced by non-health factors associated with sickness absence-for example, related to employment (job type, security, management style, work environment), past experiences of sickness (perceptions of vulnerability) or pressures outside of work (eg, caring responsibilities, financial difficulties). ${ }^{26-29}$ While $85 \%$ of working age Lambeth residents from White ethnic groups are employed, only $66 \%$ of Blackand minority ethnic (BME) working age residents in Lambeth are in employment. ${ }^{13}$ Although unemployed patients may still require a fit note to apply for long-term sickness benefits, only a minority of fit notes are used for benefit applications. ${ }^{30}$ Higher unemployment might imply that we would find a lower risk of fit note receipt in the BME population because they are less likely in need to present sickness certification to an employer. However, employment type varies by ethnicity and national studies suggest that some ethnic groups, in particular the Black Caribbean group, are more likely to be in care-providing, low-skilled ${ }^{26}$ and precarious work. ${ }^{31}$ Lowskilled work is associated with low job control, lower satisfaction, financial difficulties and increased sickness absence. ${ }^{32-34}$ Precarious employment has been found to be associated with higher injury risks, disease risk, hazard exposures and lower worker and manager knowledge of occupational health services (OHS) and regulatory responsibilities. ${ }^{33}$ Employers with less awareness of OHS may be more likely to request a fit note before the seven official days and less likely to adapt to employees during periods of illness, leading unwell employees to require longer periods of sickness absence which entail fit note use. ${ }^{2}$ Unsupportive, demanding and precarious employment, coupled with higher risks of long-term conditions, may contribute to the increased risk of FFN receipt among the Black African, Black Caribbean and BlackOther populations which we find in this study.

We demonstrate the relative contribution of different longterm conditions to the risk of fit note receipt. While virtually all long-term conditions were associated with increased risk of fit note receipt, the largest increases were observed for chronic pain and depression. Consistent with the international literature on the enormous impact of depression on work, ${ }^{38-40}$ depression had the highest overall PAF for FFN use indicating that in population terms it is the most important diagnosis in determining fit note use. Obesity was also observed to have a strong association with FFN receipt, consistent with previous work on specific occupational groups. $^{41}$ 


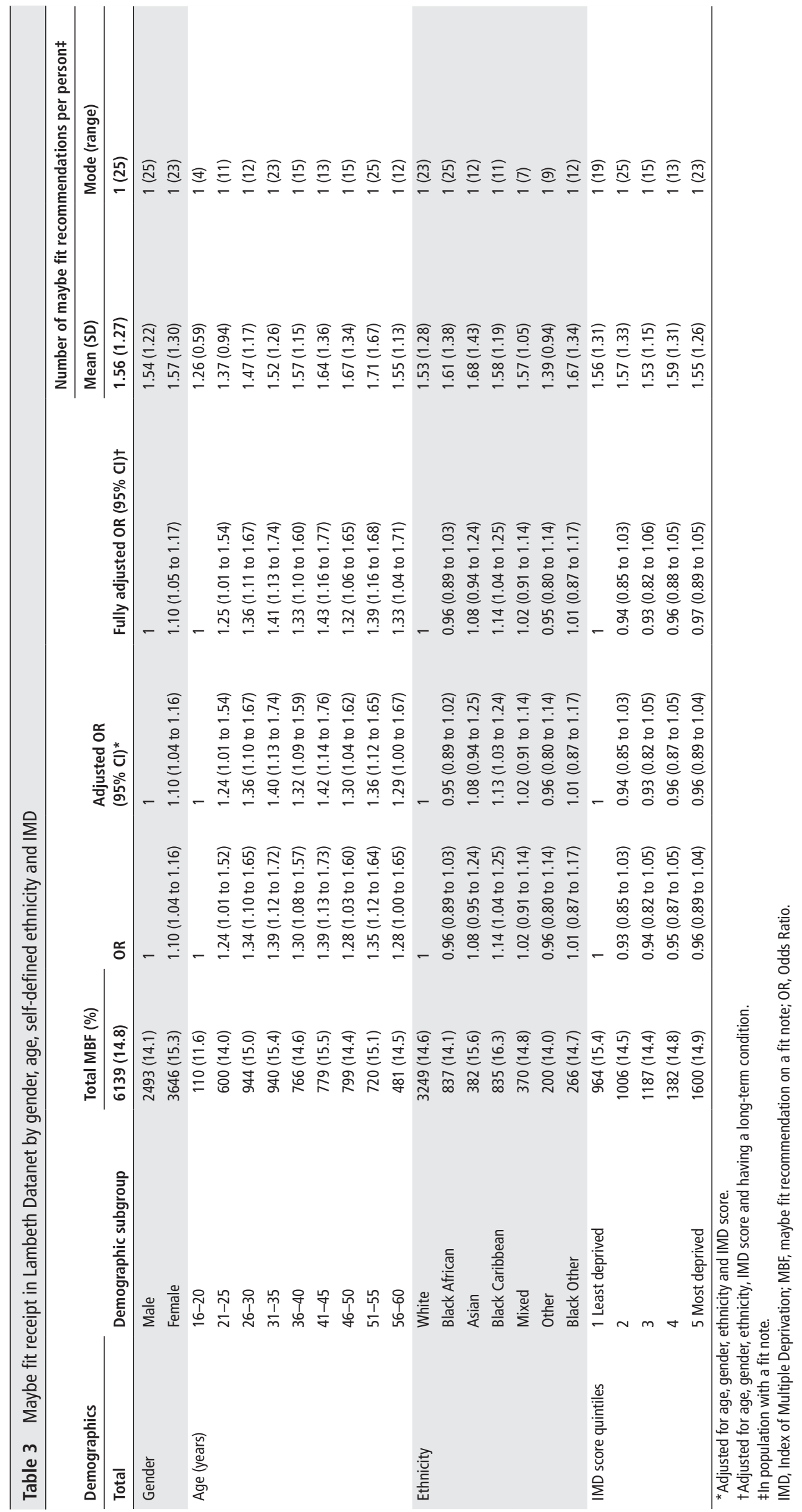


Table 4 Risk of FFN receipt by LTC stratified by age group

\begin{tabular}{|c|c|c|c|c|c|c|}
\hline LTC; by age in years & N (\%) 326415 & N people with FFN since 2014 & Rate FFN per 1000 & $\mathrm{HR}^{*}(95 \% \mathrm{Cl})$ & Adjusted hazard ratio $(95 \% \mathrm{Cl}) \dagger$ & PAF§ \\
\hline No LTC & $212436(65.1)$ & $16647(7.8)$ & 39.1 & 1 & 1 & $0.0 \%$ \\
\hline $16-40$ & $172445(72.9)$ & $11903(6.9)$ & 36.1 & 1 & 1 & $0.0 \%$ \\
\hline $41-50$ & $28130(50.3)$ & $3136(11.2)$ & 46.9 & 1 & 1 & $0.0 \%$ \\
\hline $51-60$ & $11861(35.0)$ & $1608(13.6)$ & 56.0 & 1 & 1 & $0.0 \%$ \\
\hline Depression & 33477 (10.3) & $10271(30.7)$ & 155.8 & 3.9 (3.7 to 4.1$)$ & 3.4 (3.3 to 3.6$)$ & $17.5 \%$ \\
\hline $16-40$ & $19312(8.2)$ & $5060(26.2)$ & 141.3 & $3.8(3.7$ to 4.0$)$ & 3.6 (3.5 to 3.8$)$ & $15.3 \%$ \\
\hline $41-50$ & $8312(14.9)$ & $2965(35.7)$ & 168 & 3.5 (3.3 to 3.7$)$ & 3.3 (3.1 to 3.5$)$ & $19.6 \%$ \\
\hline $51-60$ & $5853(17.2)$ & $2246(38.4)$ & 179.7 & 3.1 (2.8 to 3.3$)$ & 2.9 (2.7 to 3.2$)$ & $18.2 \%$ \\
\hline Cancer & $3557(1.1)$ & $1138(32.0)$ & 150.5 & 3.8 (3.4 to 4.2$)$ & $3.0(2.7$ to 3.3$)$ & $1.8 \%$ \\
\hline $16-40$ & $1050(0.4)$ & $231(22.0)$ & 110.8 & $3.0(2.6$ to 3.5$)$ & 3.1 (2.7 to 3.5$)$ & $0.7 \%$ \\
\hline $41-50$ & $998(1.8)$ & $386(38.7)$ & 182.4 & 3.8 (3.3 to 4.4$)$ & $3.5(3.1$ to 4.0$)$ & $2.6 \%$ \\
\hline $51-60$ & $1509(4.5)$ & $521(34.5)$ & 155 & 2.7 (2.4 to 3.0$)$ & 2.5 (2.2 to 2.9 ) & $3.9 \%$ \\
\hline Obesity & $49847(15.3)$ & $11821(23.7)$ & 108.6 & 2.7 (2.5 to 2.9$)$ & 2.1 (2.0 to 2.2$)$ & $14.9 \%$ \\
\hline $16-40$ & 23396 (9.9) & 4356 (18.6) & 90.3 & 2.5 (2.3 to 2.7$)$ & 2.0 (1.9 to 2.2$)$ & $9.1 \%$ \\
\hline $41-50$ & $14594(26.1)$ & $3915(26.8)$ & 117.4 & 2.5 (2.3 to 2.7$)$ & 2.1 (2.0 to 2.2 ) & $19.4 \%$ \\
\hline $51-60$ & $11857(34.9)$ & $3550(29.9)$ & 130.3 & $2.3(2.1$ to 2.5$)$ & 2.1 (1.9 to 2.2$)$ & $23.0 \%$ \\
\hline Diabetes‡ & $9.946(3.1)$ & $2830(28.5)$ & 128.8 & 3.2 (2.9 to 3.6$)$ & 2.2 (2.1 to 2.4$)$ & $3.7 \%$ \\
\hline $16-40$ & $2036(0.9)$ & $408(20.0)$ & 101.6 & $2.8(2.5$ to 3.1$)$ & $2.4(2.1$ to 2.7$)$ & $1.0 \%$ \\
\hline $41-50$ & $3258(5.8)$ & 940 (28.9) & 126.6 & $2.7(2.4$ to 3.0$)$ & 2.2 (2.0 to 2.5$)$ & $4.9 \%$ \\
\hline $51-60$ & $4652(13.7)$ & $1482(31.9)$ & 140.6 & 2.5 (2.2 to 2.7$)$ & 2.3 (2.1 to 2.5$)$ & $10.4 \%$ \\
\hline SMI & $4598(1.4)$ & $1227(26.7)$ & 126.8 & 3.2 (2.9 to 3.5$)$ & 2.4 (2.2 to 2.6$)$ & $1.7 \%$ \\
\hline $16-40$ & $2169(0.9)$ & $615(28.4)$ & 154.1 & 4.2 (3.8 to 4.7 ) & 3.3 (3.0 to 3.7 ) & $1.8 \%$ \\
\hline $41-50$ & $1357(2.4)$ & $376(27.7)$ & 122.6 & 2.6 (2.3 to 3.0$)$ & 2.1 (1.9 to 2.4 ) & $1.9 \%$ \\
\hline $51-60$ & $1072(3.2)$ & $236(22.0)$ & 90.1 & 1.6 (1.4 to 1.9$)$ & $1.4(1.2$ to 1.7$)$ & $0.8 \%$ \\
\hline Cardio & $1144(0.4)$ & 379 (31.4) & 147.6 & 3.7 (3.3 to 4.2 ) & 2.8 (2.5 to 3.3$)$ & $0.6 \%$ \\
\hline $16-40$ & $211(0.1)$ & $49(23.2)$ & 117.3 & 3.2 (2.4 to 4.3$)$ & 2.8 (2.1 to 3.7$)$ & $0.1 \%$ \\
\hline $41-50$ & $331(0.6)$ & $117(35.4)$ & 175.8 & 3.7 (3.0 to 4.5$)$ & 3.3 (2.7 to 4.1$)$ & $0.8 \%$ \\
\hline $51-60$ & $602(1.8)$ & $193(32.1)$ & 143.1 & 2.5 (2.1 to 3.0$)$ & 2.5 (2.1 to 3.0$)$ & $1.4 \%$ \\
\hline Vascular & $1474(0.5)$ & $534(36.2)$ & 172.5 & 4.3 (3.9 to 4.8$)$ & 3.0 (2.7 to 3.4$)$ & $0.9 \%$ \\
\hline $16-40$ & $203(0.1)$ & $62(30.5)$ & 151.1 & 4.1 (3.1 to 5.5$)$ & 3.4 (2.5 to 4.6$)$ & $0.2 \%$ \\
\hline $41-50$ & $408(0.7)$ & $169(41.4)$ & 212.6 & 4.4 (3.7 to 5.3 ) & 3.7 (3.0 to 4.5$)$ & $1.2 \%$ \\
\hline $51-60$ & $863(2.5)$ & $303(35.1)$ & 160.2 & 2.8 (2.4 to 3.2$)$ & 2.6 (2.3 to 3.0$)$ & $2.5 \%$ \\
\hline Respiratory & $35153(10.8)$ & $6156(17.5)$ & 85.0 & 2.2 (2.1 to 2.3$)$ & $2.0(1.9$ to 2.0$)$ & $7.4 \%$ \\
\hline $16-40$ & $25188(10.7)$ & $3214(12.8)$ & 65.0 & 1.8 (1.7 to 1.9$)$ & $1.7(1.7$ to 1.8$)$ & $5.5 \%$ \\
\hline $41-50$ & $5828(10.4)$ & $1566(26.9)$ & 116.4 & 2.4 (2.3 to 2.6$)$ & $2.3(2.1$ to 2.4$)$ & $8.4 \%$ \\
\hline $51-60$ & $4137(12.2)$ & $1376(33.3)$ & 147.4 & 2.6 (2.3 to 2.8 ) & 2.3 (2.1 to 2.5$)$ & $9.6 \%$ \\
\hline Chronic pain & $4550(1.4)$ & $1870(41.1)$ & 201.3 & $5.0(4.5$ to 5.5$)$ & 3.7 (3.3 to 4.0 ) & $3.3 \%$ \\
\hline $16-40$ & $990(0.4)$ & $412(41.6)$ & 221.1 & 6.0 (5.2 to 6.8$)$ & 4.8 (4.3 to 5.5$)$ & $1.4 \%$ \\
\hline $41-50$ & $1568(2.8)$ & $671(42.8)$ & 212.3 & 4.4 (3.9 to 4.9 ) & 3.7 (3.4 to 4.1$)$ & $4.6 \%$ \\
\hline $51-60$ & $1992(5.9)$ & 787 (39.5) & 184.4 & 3.2 (2.8 to 3.6$)$ & 2.9 (2.6 to 3.3$)$ & $6.4 \%$ \\
\hline LD & $839(0.3)$ & $170(20.3)$ & 81.4 & 2.1 (1.6 to 2.6 ) & 1.5 (1.2 to 1.8$)$ & $0.1 \%$ \\
\hline $16-40$ & $432(0.2)$ & $92(21.3)$ & 89.0 & 2.4 (1.9 to 3.2$)$ & 1.8 (1.4 to 2.4$)$ & $0.2 \%$ \\
\hline $41-50$ & $229(0.4)$ & $48(21.0)$ & 84.1 & 1.8 (1.3 to 2.4$)$ & 1.4 (1.0 to 1.8$)$ & $0.1 \%$ \\
\hline $51-60$ & $178(0.5)$ & $30(16.9)$ & 62.1 & 1.1 (0.8 to 1.5$)$ & $1.0(0.7$ to 1.3$)$ & $0.0 \%$ \\
\hline Epilepsy & $2298(0.7)$ & $574(25.0)$ & 117.9 & 3.0 (2.7 to 3.3$)$ & 2.6 (2.3 to 2.9$)$ & $0.9 \%$ \\
\hline $16-40$ & $1254(0.5)$ & $266(21.2)$ & 107.3 & 2.9 (2.6 to 3.3$)$ & 2.8 (2.5 to 3.2$)$ & $0.7 \%$ \\
\hline $41-50$ & $583(1.0)$ & $170(29.2)$ & 130.5 & 2.7 (2.3 to 3.3 ) & 2.5 (2.0 to 3.0$)$ & $1.0 \%$ \\
\hline $51-60$ & 461 (1.4) & $138(29.9)$ & 127.3 & 2.2 (1.9 to 2.7$)$ & 2.2 (1.8 to 2.6$)$ & $0.9 \%$ \\
\hline RA & $827(0.3)$ & $258(31.2)$ & 146.5 & 3.7 (3.3 to 4.1$)$ & $2.6(2.3$ to 3.0$)$ & $0.4 \%$ \\
\hline $16-40$ & $257(0.1)$ & 58 (22.6) & 111.6 & $3.1(2.4$ to 4.0$)$ & 2.6 (2.0 to 3.5$)$ & $0.2 \%$ \\
\hline $41-50$ & $243(0.4)$ & 79 (32.5) & 148.5 & 3.1 ( 2.5 to 3.9 ) & 2.7 (2.1 to 3.4$)$ & $0.5 \%$ \\
\hline $51-60$ & $327(1.0)$ & $121(37.0)$ & 170.6 & 3.0 (2.4 to 3.7$)$ & 2.6 (2.0 to 3.2 ) & $0.9 \%$ \\
\hline Hypertension & $18190(5.6)$ & $5400(29.7)$ & 131.6 & 3.3 (3.1 to 3.5 ) & 2.2 (2.1 to 2.4 ) & $7.1 \%$ \\
\hline $16-40$ & $2765(1.2)$ & $642(23.2)$ & 110.1 & 3.0 (2.7 to 3.3$)$ & 2.3 (2.2 to 2.5$)$ & $1.5 \%$ \\
\hline $41-50$ & $6270(11.2)$ & $1867(29.8)$ & 130.7 & 2.8 (2.5 to 3.0$)$ & 2.2 (2.1 to 2.4$)$ & $9.6 \%$ \\
\hline $51-60$ & $9155(27.0)$ & 2891 (31.6) & 138.2 & 2.4 (2.2 to 2.6$)$ & 2.2 (2.0 to 2.4$)$ & $19.5 \%$ \\
\hline
\end{tabular}

*Hazard ratios estimate a ratio of time to FFN for groups with a LTC compared with the group with no LTC.

tAdjusted for age, sex, ethnicity and deprivation.

‡Diabetes type 2 ;

\$PAF estimates the proportion of the incidence of fit note receipt that is potentially attributable to having the LTC

Cardio, coronary heart disease, atrial fibrillation and heart failure; FFN, first fit note; LD, disability; LTC, long-term condition; PAF, population attributable fraction; RA, rheumatoid arthritis; SMI, serious mental illness;

Vascular, peripheral arterial disease, stroke and transient ischaemia. 
Table 5 Maybe fit recommendation among people who receive a fit note by LTC

\begin{tabular}{|c|c|c|c|c|}
\hline LTC & N people with FFN since 2014 & Number of MBF (\%) & OR $(95 \% \mathrm{Cl})$ & Adjusted $\mathrm{OR}^{*}(95 \% \mathrm{Cl})$ \\
\hline No LTC & 16647 & $2446(14.7)$ & 1.00 & 1.00 \\
\hline Depression & 10271 & $1479(14.4)$ & 0.97 (0.91 to 1.05$)$ & 0.96 (0.89 to 1.03 ) \\
\hline Obesity & 11821 & $1823(15.4)$ & 1.05 (0.98 to 1.13$)$ & 1.04 (0.97 to 1.13$)$ \\
\hline Respiratory & 6156 & $924(15.0)$ & $1.02(0.92$ to 1.14$)$ & 1.01 (0.91 to 1.13$)$ \\
\hline Diabetest & 2830 & $385(13.6)$ & $0.91(0.81$ to 1.03$)$ & $0.88(0.77$ to 1.00$)$ \\
\hline Vascular & 534 & $84(15.7)$ & 1.08 (0.85 to 1.37$)$ & 1.04 (0.82 to 1.32 ) \\
\hline Chronic pain & 1870 & $274(14.7)$ & 0.99 (0.87 to 1.13$)$ & 0.97 (0.85 to 1.12$)$ \\
\hline Cancer & 1138 & $180(15.8)$ & 1.09 (0.93 to 1.27$)$ & 1.05 (0.89 to 1.26$)$ \\
\hline Epilepsy & 574 & 65 (11.3) & 0.74 (0.58 to 0.94$)$ & 0.73 (0.56 to 0.94$)$ \\
\hline Rheumatoid arthritis & 258 & $29(11.2)$ & $0.73(0.47$ to 1.14$)$ & 0.70 (0.45 to 1.10$)$ \\
\hline Learning disability & 170 & $17(10.0)$ & 0.64 (0.40 to 1.03$)$ & 0.64 (0.40 to 1.02$)$ \\
\hline
\end{tabular}

${ }^{*}$ Adjusted for age, sex, ethnicity and deprivation.

tDiabetes type 2.

Cardio, coronary heart disease, atrial fibrillation and heart failure ; FFN, first fit note; LTC, long-term condition; MBF, maybe fit recommendation on a fit note; OR, Odds Ratio; SMI, serious mental illness ; Vascular, peripheral arterial disease, stroke and transient ischaemia.

We note relatively low use of 'maybe fit' recommendations which are in effect a voluntary system to encourage employers and employees to consider adjustments. The lack of legal status of 'maybe fit' may contribute to its low use. National studies have found 'maybe fit' recommendations to be higher in areas of lower deprivation. We did not find trends in 'maybe fit' use by area-level deprivation, but consistent with previous studies we found 'maybe fit' use was higher among women. ${ }^{6}$ Previous studies have found that individuals with mental disorders, including fit note prescriptions for stress and low mood, are associated with reduced 'maybe fit' use. ${ }^{6}$ We found SMI and epilepsy were associated with reduced 'maybe fit' use. This might suggest that the doctors prescribing the fit note view occupational impairments resulting from such diagnoses as relatively immutable. By contrast, depression was not associated with 'maybe fit' use.

\section{Limitations}

While our analysis of complete population data at an individuallevel (rather than aggregated) improves on previous data sets used in this field, there are inevitable limitations in using such routine clinical data. We were limited to a single geographical area for which we had GP data. This had the benefit of providing information on virtually all individuals seeking help within that population, but it had the disadvantage of a possible loss of generalisability, and our findings should be tested in other areas. While we included all patients who were registered with GPs in Lambeth, this is not the same as all people residents in Lambeth-some of the individuals in our study lived outside the Borough's boundaries but they were registered with GPs in Lambeth, and it is likely that some Lambeth residents were not included because they were registered with GPs from neighbouring boroughs. As the neighbouring populations are somewhat similar and this is likely to affect only the population at the boundaries of the borough, we suggest that this is unlikely to impact our main findings. We were unable to explore contextual factors such as the nature of employment, educational level or benefit information, making it impossible to ascertain whether fit notes were issued for individuals in work versus those already out of work who were applying for health benefits. Furthermore, we did not know the occupational status of individuals within the population-for example whether they were unemployed, retired or in full-time education. We did not have access to sickness certification before the start of our study window, therefore our results do not represent lifetime FFN. If we were able to access information on lifetime FFN we would expect to see a shift towards younger age groups, and an increase in diagnosis, such as depression, associated with younger age groups. We could not determine the proportion of people who do not receive a fit note despite being ill-either due to relaxed work policies or because they feel unable to take time off work. Furthermore, long-term conditions were assessed using QOF which provides incomplete diagnostic information and we were unable to access information on the reason the GP gave for giving the fit note, because free text data extraction is not permitted from anonymised primary care data sets. Despite these limitations, to our knowledge, this is the largest study to analyse fit note use and long-term conditions using individual-level clinical data.

\section{IMPLICATIONS}

This paper finds wide variation in fit note use by demographic variables and long-term conditions. It also highlights gaps in our knowledge about fit note use and the challenges of studying occupational outcomes using routine health data. Our understanding would be improved if future cohort data sets and data linkages included information on fit note receipt and occupation. Research is needed to improve our knowledge of the occupational health needs of the population that the fit note is designed to support. Such analyses are required in order to evaluate the relatively new policy which fit notes represent.

\section{Author affiliations}

${ }^{1}$ Department of Psychological Medicine, Institute of Psychiatry, Psychology \& Neuroscience, King's College London, London, UK

${ }^{2}$ Biomedical Research Nucleus, South London and Maudsley Mental Health NHS Trust, London, UK

${ }^{3}$ Biostatistics and Health Informatics, King's College London, London, UK ${ }^{4}$ King's Centre for Military Health Research, King's College London, London, UK ${ }^{5}$ School of Population Health and Environmental Sciences, King's College London, London, UK

${ }^{6}$ Department of Mental Health and Suicide, Norwegian Institute of Public Health, Oslo, Norway

${ }^{7}$ Norway Centre for Work and Mental Health, Nordland Hospital Trust, Bodø, Norway ${ }^{8}$ Norway Research Unit, Directorate of Labour and Welfare, Oslo, Norway
} 
${ }^{9}$ Norway Centre for Research and Education in Forensic Psychiatry and Psychology, Haukeland University Hospital, Bergen, UK

${ }^{10}$ Department of Community Medicine, University of Tromsø, Tromsø, Norway

${ }^{11}$ Department of Occupational Health, Guys and St Thomas NHS Foundation Trust, London, UK

Acknowledgements Thanks to Megan Pritchard and Amelia Jewell at the NIHR Maudsley Biomedical Research Centre for their support with this study.

Contributors Sarah Dorrington designed the study, analysed the data and drafted the manuscript. Matthew Hotopf, Stephani Hatch and Ira Madan advised extensively throughout the process. Ewan Carr advised on the statistical analysis. Matthew Broadbent, David Whitney, Stevo Durbaba, Mark Ashworth, Sharon Stevelink and Alex Dregan provided expertise and guidance on extracting, analysing and interpreting the data. Arnstein Mykletun and all authors commented on the final manuscript.

Funding This paper represents independent research part funded by the Royal College of Psychiatrists' Donald Dean Fellowship and the National Institute for Health Research Biomedical Research Centre at South London and Maudsley NHS Foundation Trust and King's College London.

Disclaimer The views expressed are those of the authors and not necessarily those of the Royal College of Psychiatrists, the NHS, the NIHR or the Department of Health and NIHR Maudsley Biomedical Research Centre, South London and Maudsley NHS Foundation Trust, London, UK.

Competing interests Professor $\mathrm{MH}$ receives funding from Janssen as part of the RADAR-CNS consortium which includes a project on depression. He is a principal investigator of RADAR-CNS, a pre-competitive public-private partnership co-funded by Innovative Medicines Initiative (European Commission) and European Federation of Pharmaceutical Industries and Associations. He has also been an independent expert witness in group litigations instructed by claimants against pharmaceutical companies for alleged harmful effects of their products. The authors have no other conflict of interest to declare.

Patient consent for publication Not required.

Ethics approval CRIS was established in 2008 and approved by the Oxfordshire Research Ethics Committee in 2008 (reference 18/SC/0372). Approval for linkage with Lambeth DataNet was granted by Lambeth Clinical Commissioning Group and Information Governance Committee. This project was approved by the CRIS Oversight Committee in 2015.

Provenance and peer review Not commissioned; externally peer reviewed.

Data availability statement Data may be obtained from a third party and are not publicly available. Access to the deidentified data in this study can be applied for through Lambeth Datanet and South London and Maudsley Biomedical Research Centre (BRC), De Crespigny Park, London SE5 8AF (cris.administrator@slam.nhs.uk). Access is granted only if approval is given by both the BRC and the Lambeth Datanet Steering Group.

\section{ORCID iDs}

Sarah Dorrington http://orcid.org/0000-0002-6462-1880

Ewan Carr http://orcid.org/0000-0002-1146-4922

Sharon A M Stevelink http://orcid.org/0000-0002-7655-7986

Ira Madan http://orcid.org/0000-0003-2200-7329

Stephani Hatch http://orcid.org/0000-0001-9103-2427

Matthew Hotopf http://orcid.org/0000-0002-3980-4466

\section{REFERENCES}

1 Henderson M, Clark C, Stansfeld S, et al. A lifecourse approach to long-term sickness absence - a cohort study. PLoS One 2012;7:e36645.

2 Black CM. Working for a healthier tomorrow: Dame Carol Black's review of the health of Britain's working age population. London: TSO, 2008

3 Wainwright $E$, Wainwright $D$, Keogh $E$, et al. The social negotiation of fitness for work: tensions in doctor-patient relationships over medical certification of chronic pain. Health 2015;19:17-33.

4 Health at work - an independent review of sickness absence by Dame Carol Black and David Frost C.B.E 2011

5 Kausto J, Solovieva S, Virta LJ, et al. Partial sick leave associated with disability pension: propensity score approach in a register-based cohort study. BMJ Open 2012;2. doi:10.1136/bmjopen-2012-001752. [Epub ahead of print: 0811 2012].

6 Dorrington S, Roberts E, Mykletun A, et al. Systematic review of fit note use for workers in the UK. Occup Environ Med 2018;75:530-9.

7 Chenery V. An evaluation of the statement of fitness for work (fit note): a survey of employees. Sheffield: Department for Work and Pensions, 2013.

8 Fit notes issued by GP practices: December 2014-March 2017: experimental statistics: NHS Digital 2017.
9 Shiels CH, J; Pollard E, Gabbay M. Evaluation of the statement of fitness for work (fit note): quantitative survey of fit notes. Research report 2013. (no 841):95 pages.

10 Coole C, Watson PJ, Thomson L, et al. How do GPs complete fit note comments? Occup Med 2013:63:575-8.

11 Lambeth Datanet Community Engagement Project. Healthwatch Lambeth, 2017.

12 Kumarapeli P, Stepaniuk R, de Lusignan S, et al. Ethnicity recording in general practice computer systems. J Public Health 2006;28:283-7.

13 Lambeth Council: state of the borough Lambeth Council 2016.

14 Trust for London: Tackling poverty and inequality. Overview of London boroughs, 2019. https://www.trustforlondon.org.uk/data/boroughs/overview-of-london-boroughs

15 Mixed and balanced communities: census information service, greater London authority, London 2014

16 LSOA Atlas. Greater London authority, 2014. Available: https://data.london.gov.uk/ dataset/lsoa-atlas

17 Hatch SL, Frissa S, Verdecchia M, et al. Identifying socio-demographic and socioeconomic determinants of health inequalities in a diverse London community: the South East London community health (SELCOH) study. BMC Public Health 2011;11:861.

18 Census Analysis. Ethnicity and religion of the non-UK born population in England and Wales: 2011, 2011. Available: https://www.ons.gov.uk/peoplepopulationandc ommunity/culturalidentity/ethnicity/articles/2011censusanalysisethnicityandreligionof thenonukbornpopulationinenglandandwales/2015-06-18\#ethnicity-of-the-non-ukborn-population [Accessed August 2019].

19 QOF Business Rules—version 35. Department of health , 2017

20 From one to many: exploring people's progression to multiple long-term conditions in an urban environment, 2018. Available: https://www.gsttcharity.org.uk/what-wedo/our-programmes/multiple-long-term-conditions/one-many-exploring-peoplesprogression

21 The King's Fund. Impact of quality and outcomes framework on health inequalities 2011.

22 Economou A, Grey M, McGregor J, et al. The health informatics cohort enhancement project (HICE): using routinely collected primary care data to identify people with a lifetime diagnosis of psychotic disorder. BMC Res Notes 2012;5:95.

23 Mansournia MA, Altman DG. Population attributable fraction. BMJ 2018;360:k757.

24 Schofield P, Saka O, Ashworth M. Ethnic differences in blood pressure monitoring and control in South East London. Br J Gen Pract 2011;61:e190-6.

25 Nazroo JY, Falaschetti E, Pierce M, et al. Ethnic inequalities in access to and outcomes of healthcare: analysis of the health survey for England. J Epidemio/ Community Health 2009;63:1022-7.

26 Employment by Occupation. Uk government, 2018. Available: https://www.ethnicityfacts-figures.service.gov.uk/work-pay-and-benefits/employment/employment-byoccupation/latest

27 Jansen NWH, Kant IJ, van Amelsvoort LGPM, et al. Work-family conflict as a risk factor for sickness absence. Occup Environ Med 2006:63:488-94.

28 Milligan-Saville JS, Tan L, Gayed A, et al. Workplace mental health training for managers and its effect on sick leave in employees: a cluster randomised controlled trial. Lancet Psychiatry 2017;4:850-8.

29 Harvey SB, Wang M-J, Dorrington S, et al. NIPSA: a new scale for measuring nonillness predictors of sickness absence. Occup Environ Med 2018;75:98-104.

30 Stat-Xplore department for work and pensions, department of health. Available: https:// stat-xplore.dwp.gov.uk/webapi/jsf/tableView/tableView.xhtml [Accessed Sept 2019].

31 Insecure work and ethnicity. London: Trades Union Congress 2017.

32 Schrijvers CT, van de Mheen HD, Stronks K, et al. Socioeconomic inequalities in health in the working population: the contribution of working conditions. Int J Epidemiol 1998:27:1011-8

33 Quinlan M, Mayhew C, Bohle P. The global expansion of precarious employment, work disorganization, and consequences for occupational health: a review of recent research. Int J Health Serv 2001;31:335-414.

34 North F, Syme SL, Feeney A, et al. Explaining socioeconomic differences in sickness absence: the Whitehall II study. BMJ 1993;306:361-6.

35 Moscone F, Tosetti E, Vittadini G. The impact of precarious employment on mental health: the case of Italy. Soc Sci Med 2016;158:86-95.

36 Matilla-Santander N, Lidón-Moyano C, González-Marrón A, et al. Measuring precarious employment in Europe 8 years into the global crisis. J Public Health 2019;41:259-67.

37 Harvey SB, Modini M, Joyce S, et al. Can work make you mentally ill? A systematic meta-review of work-related risk factors for common mental health problems. Occup Environ Med 2017:74:301-10.

38 Kessler RC, Frank RG. The impact of psychiatric disorders on work loss days. Psychol Med 1997;27:861-73.

39 Henderson M, Harvey SB, Øverland S, et al. Work and common psychiatric disorders. J $R$ Soc Med 2011:104:198-207.

40 Knudsen AK, Harvey SB, Mykletun A, et al. Common mental disorders and long-term sickness absence in a general working population. The Hordaland health study. Acta Psychiatr Scand 2013;127:287-97.

41 Harvey SB, Glozier N, Carlton 0, et al. Obesity and sickness absence: results from the CHAP study. Occup Med 2010;60:362-8. 